\title{
RPAS COMMUNICATION CHANNELS BASED ON WCDMA 3GPP STANDARD
}

\author{
Andrii GREKHOV (D) ${ }^{1, *}$, Vasyl KONDRATIUK ${ }^{2}$, Svitlana ILNYTSKA ${ }^{3}$ \\ ${ }^{1}$ Air Navigation Systems Department, National Aviation University, Kyiv, Ukraine \\ ${ }^{2}$ Research and Training Centre "Aerospace Centre", National Aviation University, Kyiv, Ukraine \\ ${ }^{3}$ Institute of Laser and Optoelectronics Intelligent Manufacturing Wenzhou University, Wenzhou, China
}

Received 11 March 2019; accepted 09 February 2020

\begin{abstract}
First built models of Remotely Piloted Air System (RPAS) communication channels based on Wideband Code Division Multiple Access (WCDMA) 3GPP Standard were designed. Base Station (BS) transmission within the Radio Line of Sight (RLoS) and through the satellite using Beyond Radio Line of Sight (BRLoS) was considered. The dependencies of the Bit Error Rate (BER) on the signal-noise ratio for different RPAS velocities and WCDMA channel models were obtained. The dependences of the BER on the signal-noise ratio for different levels of satellite transponder nonlinearity were studied. The dependence of the BER on the BS antenna diameter in case of the transponder nonlinearity was analysed. The dependencies for satellite channel characteristics, first obtained taking into account the motion of the RPAS, make it possible to predict the behavior of the communication system in critical conditions.
\end{abstract}

Keywords: RPAS, satellite links, WCDMA, data transmission, transmitter nonlinearity, RPAS speed.

\section{Introduction}

Advances in technology of the Remotely Piloted Air System (RPAS) or the Unmanned Aerial Vehicle (UAV) led to the creation of RPASs with various sizes, which have on board enhanced processing, memory, data storage and communication capabilities. RPASs are extensively used nowadays in different military applications, surveillance and monitoring, telecommunications, agriculture, search and rescue operations, fire detection, exploration of volcanic environments, power line and structure inspection, natural disaster recover, data collection from the wireless sensor networks (Liu et al., 2018; Mozaffari et al., 2019).

RPASs must effectively communicate with each other and with existing network infrastructures. Compared with the widely used cellular or satellite systems, the RPAS communication has its own distinctive channel characteristics (Gupta, Jain, \& Vaszkun, 2015). Accurate channel operation is critical to optimize performance and design for effective RPAS communications (Zeng, Zhang, \& Lim, 2016a). There is a problem in modeling the RPAS communication channel, because the propagation characteristics of the RPAS channels are not well understood for spatial and temporal changes in non-stationary channels. In the paper (Imad et al., 2017) the functions, services and requirements for communication systems based on RPASs are defined. Network architectures, basic structures and data traffic requirements in these systems, as well as the use of RPASs for collecting data from wireless sensor networks are also presented. In the article (Khuwaja et al., 2018) approaches to RPAS communication channel modeling and determining channel characteristics are considered.

The paper (Zeng, Zhang, \& Lim, 2016b) reviewed a new mobile relay technique in which relay nodes are mounted on RPASs and, therefore, are able to move at high speed. Compared to conventional static relaying, mobile relaying provides a new degree of freedom to increase productivity by carefully designing the relay trajectory.

The aeronautical communications network (ACN) has attracted a great deal of attention from industry as well as scientists, due to the increasing demands on accurate surveillance services and rapid response to emergencies. ACNs are heterogeneous networks and are designed to use satellites, high-altitude platforms and low-level platforms. Compared with terrestrial wireless networks, ACNs are characterized by frequently changing network topologies and more vulnerable communication connections. Moreover, ACNs have a need for seamless integration of heterogeneous networks (Cao et al., 2018).

${ }^{*}$ Corresponding author. E-mail: grekhovam@gmail.com

This is an Open Access article distributed under the terms of the Creative Commons Attribution License (https://creativecommons.org/licenses/by/4.0/), which permits unrestricted use, distribution, and reproduction in any medium, provided the original author and source are credited. 
There is a supply/demand mismatch between the ground infrastructure and the sudden need to increase traffic. One approach to solving this problem is the use of RPAS technology. A new advanced access network architecture has been proposed (Dong et al., 2018), in which the fronthaul and backhaul links are mounted on RPASs for rapid event response and flexible deployment.

A promising technology for future communication systems is wireless communication with RPASs. The article (Zeng \& Zhang, 2017) examines the energy-efficient communication of the RPAS with the ground terminal through the optimization of the RPAS trajectory. Energy efficiency of communication with a RPAS is defined as the total number of transmitted information bits, normalized by the energy of the RPAS motion consumed over a finite time interval. A design is proposed to maximize the RPAS energy efficiency with general constraints on the trajectory, including its starting/ending locations and speeds, as well as the minimum/maximum speed and acceleration.

The RPAS communication channel was simulated using the statistical model for analyzing the performance of the data transmission channel (Bing, 2017). The channel model included large-scale and small-scale fading. Communication and networking of RPAS-based systems were considered in papers (Hanscom \& Bedford, 2016; Jawhara et al., 2017). In paper (Mozaffari et al., 2019) a tutorial on the potential benefits and applications of RPASs in wireless communications is presented. Challenges and tradeoffs in RPAS wireless networks are investigated: threedimensional deployment, performance analysis, channel modeling, and energy efficiency. Analytical frameworks and mathematical tools (optimization theory, machine learning, stochastic geometry, transport theory, and game theory) are described.

RPAS networks and drone communications are emerging areas of research with a key focus on attaining high throughput, elongated range, and enhanced coverage over the existing networks. The current state and achievements in the field of wireless communication of the RPAS are described in a paper (Sharma, 2019). Research and development related to the Flying Ad-Hoc Network (FANET) has doubled in recent years. A hybrid FANET wireless communication scheme that utilizes the capabilities of high-speed 802.11 data transmission and low power consumption of 802.15.1 was proposed in an article (Khan, Qureshi, \& Khanzada, 2019). The proposed scheme reduces the cost of communication and improves network performance in terms of bandwidth and latency.

The work (Yan, Fu, Zhang, \& Wang, 2019) examines the air-ground, ground-ground, and air-air UAV communication channels, as well as modeling these channels in various scenarios. The control of the budget of the RPAS communication line was studied taking into account losses in the line and channel attenuation effects. The spatial multiplexing during reception and transmission, which is achieved using the RPAS communication with several antennas, is analyzed. Survey on RPAS cellular communications with practical aspects, standardization advancements, regulation, and security challenges are given in (Fotouhi et al., 2019).

$5 \mathrm{G}$ mobile communications will lead to an increase in the use of RPASs, will require greater capacity and increased data transfer speed, less latency and a more flexible and scalable network. The Internet of things is one such use case. Hybrid terrestrial satellite networks and the role of RPAS in their deployment are discussed in (Marchese, Moheddine, \& Patrone, 2019).

Problems of RPAS navigation system development and the practical implementation were considered in paper (Kutsenko et al. 2017). The results of satellite communication channels simulation were published in articles (Grekhov, Kondratiuk, \& Ilnytska, 2018; Grekhov et al., 2019) and summarized in a monograph (Grekhov, 2019).

Wideband Code Division Multiple Access (WCDMA) is an example of multiple access where multiple transmitters can send information simultaneously over a single communication channel. The rapid grown of drones' market is expected in the nearest time, which is supported by analysis and forecasts of corresponding American and European bodies. The Federal Aviation Administration (FAA) projects to have 451,800 units of small commercial, 2.4 million units of small model hobbyist UAV fleet and 301,000 of remote pilots by 2022. The SESAR Joint Undertaking, in turn, predicts to have more than 7 million consumer UAVs operating across Europe in 2050 in its "European Drones Outlook Study".

Drones' market experiencing the tremendous growth now and there are some new research directions that becoming the new trends. Among the emerging research areas and potential applications, the most attractive are UAVs use in the fifth generation wireless networks, due to benefits in terms of improved coverage, less implementation costs, less latency and more reliability. Another attractive use of RPASs are Internet of Things applications and combination of RPASs in swarms. At the same time, in the literature there are no models of RPAS satellite channels based on the WCDMA 3GPP standard. The transmission of data from the Base Station (BS) within the RLoS and through the satellite using BRLoS taking into account the movement of the RPAS was not considered. There are no characteristics of the satellite channel, which allow predicting the behavior of the communication system in critical conditions.

The tasks of this of this research work: 1) to build models of RPAS communication channels based on WCDMA 3GPP Standard, including both BS transmission within the RLoS, and through the satellite using BRLoS using MATLAB software; 2) to obtain the dependencies of the Bit Error Rate (BER) on the signal-noise rate for different RPAS velocities and WCDMA RLoS channel models; 3) to obtain the dependencies of the BER on the signal-noise ratio for different levels of satellite transponder nonlinearity; 4) to study the dependence of the BER on the BS antenna diameters in case of the transponder nonlinearity. 


\section{BS-RPAS communication channel simulation}

The model of the "Base Station-to-RPAS" link (Figure 1) was designed on the base of MATLAB example "WCDMA End-to-End OPhysical Layer" commwcdmaphlayer (MATLAB, 2016) which was modified by adding in Base Station Transmitter block and in RPAS Receiver block Dish Antenna Gains. This model shows part of the frequency division duplex downlink physical layer of the third generation wireless communication system known as WCDMA. The model has the following main subsystems: Base Station Transmitter (WCDMA DL Tx block with the data channel coding scheme, WCDMA Tx Physical Mapping block, WCDMA BS Tx Antenna block, and Dish Antenna Gains block); WCDMA Channel Model block (Figure 2), and RPAS Receiver (WCDMA UE Rx Antenna block, WCDMA Rx Physical Channel Demapping and WCDMA DL Rx Channel Decoding Scheme subsystem). A channel with Additive White Gaussian Noise (AWGN) is modeled and, if selected, different channels with multipath propagation. A multipath channel through which a radio wave is transmitted can be viewed as the transmission of an initial pulse through a series of multipath components. Detailed model description can be found in example "WCDMA End-to-End Physical Layer" commwcdmaphlayer. The Transmitter (Receiver) Dish Antenna Gain block multiplies the input by a constant value (gain). The relationship between the antenna gain $\mathrm{G}$ and the antenna diameter $\mathrm{D}$ and the wavelength is determined by the relation $\mathrm{G}=\eta \cdot(\pi \mathrm{D} / \lambda)^{2}$, where $\eta$ is the antenna efficiency. The following model parameters were set up for calculations: $\eta=1$, RPAS antenna gain was taken 1.55 (an antenna diameter $\approx 0.2 \mathrm{~m}$ at $1 \mathrm{GHz}$ ), Base Station antenna gain was taken 7.8 (an antenna diameter $\approx 1.0 \mathrm{~m}$ at $1 \mathrm{GHz}$ ). The channel model is shown in Figure 2, channel types and their characteristics are given in Table 1.

The Multipath Rayleigh Fading Channel block implements a baseband simulation of a multipath Rayleigh fading propagation channel. The relative motion of the RPAS causes Doppler shifts of the signal frequency, which are set for each channel type in Table 1. For all cases, the Jakes Doppler spectrum was selected.

A multipath channel reflects signals at multiply places, therefore transmitted signal travels to the receiver along several paths. Rx finger power in Table 1 specifies the gain for each path. Finger phases in Table 1 specify the time delay for each path.

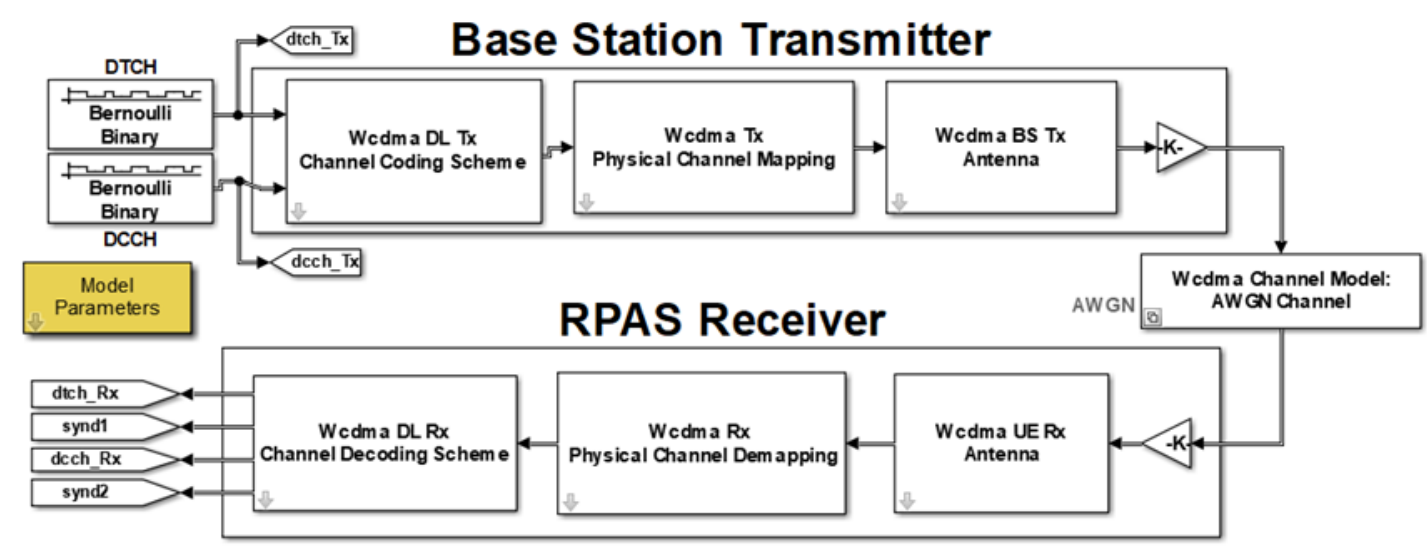

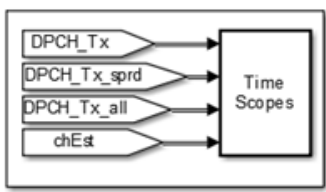

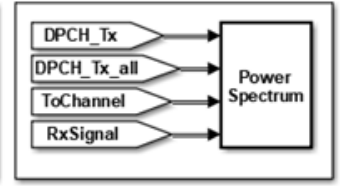

Open Scopes Close Scopes

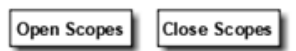

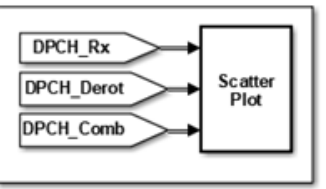

Open Scopes Close Scopes

BER/Block Error Rate (BLER)

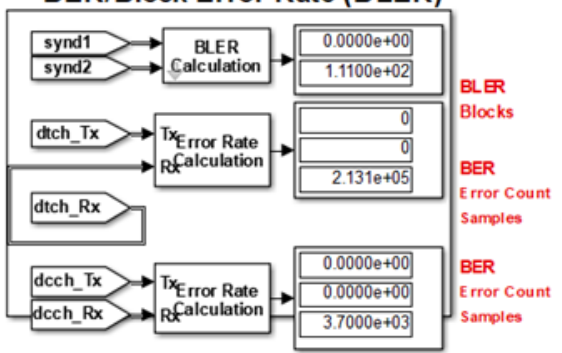

Figure 1. "Base Station-to-RPAS” link

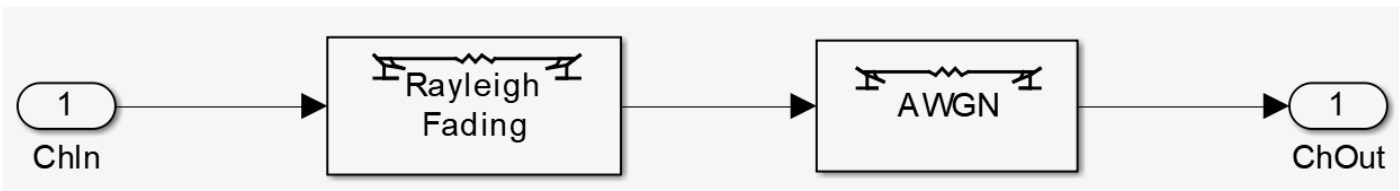

Figure 2. Channel model 
Table 1. Channels types and their characteristics (the access speed is $384 \mathrm{Kbps}$ for all cases)

\begin{tabular}{|c|c|c|c|c|c|}
\hline \multirow{2}{*}{ Channel type } & \multirow{2}{*}{$\begin{array}{l}\text { RPAS velocity } \\
(\mathrm{km} / \mathrm{h})\end{array}$} & \multirow{2}{*}{ Data channel code } & $\mathrm{R}_{\mathrm{x}}$ finger power $(\mathrm{dB})$ & \multirow{2}{*}{ Slot format } & \multirow{2}{*}{ Spreading factor } \\
\hline & & & Finger phases (s) & & \\
\hline AWGN & 0 & 15 & $\begin{array}{l}0 \\
0\end{array}$ & 14 & 16 \\
\hline Case 1 & 3 & 31 & 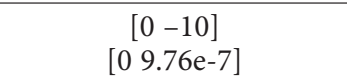 & 13 & 32 \\
\hline Case 2 & 3 & 7 & $\begin{array}{c}{\left[\begin{array}{lll}0 & 0 & 0\end{array}\right]} \\
{\left[\begin{array}{lll}0 & 9.76 \mathrm{e}-7 & 2.0 \mathrm{e}-5\end{array}\right]}\end{array}$ & 15 & 8 \\
\hline Case 3 & 120 & 31 & 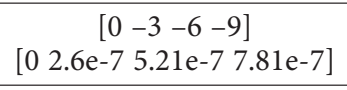 & 13 & 32 \\
\hline Case 4 & 3 & 7 & $\begin{array}{c}{\left[\begin{array}{ll}0 & 0\end{array}\right]} \\
{\left[\begin{array}{ll}0 & 9.76 \mathrm{e}-7\end{array}\right]}\end{array}$ & 15 & 8 \\
\hline Case 5 & 50 & 7 & 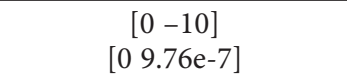 & 15 & 8 \\
\hline Case 6 & 250 & 7 & 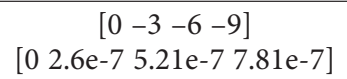 & 15 & 8 \\
\hline
\end{tabular}

WCDMA uses Direct Sequence spreading, where spreading process is done by directly combining the baseband information to high chip rate binary code. The Spreading factor in Table 1 numerically is defined as the ratio of the bandwidth in the radio channel to the speed of information transfer. The Spreading factor is an indicator characterizing the degree of redundancy of the extended frequency band relative to the spectrum of the information signal. The results of simulations are presented in Figures 3-6.

Simulation results of channels operation are given in Figure 3, which shows the dependence of the BER on the signal-noise ratio for all cases from Table 1. These data characterise the quality of data transmission over the channel for different RPAS speed. From Table 1 it can be seen that the static case $(\mathrm{v}=0 \mathrm{~km} / \mathrm{h})$ corresponds to the AWGN channel without fading. Only in this case, as can be seen from Figure 3, data transmission is carried out with the least amount of errors.

The low RPAS movement speed $(\mathrm{v}=3 \mathrm{~km} / \mathrm{h})$ corresponds to Cases 1, 2, and 4 in Table 1 . In Case 2, the channel is closed for the entire range of signal-noise values shown in Figure 3. But the data for Cases 1 and 4 show that, despite the low speed of the RPAS, the number of errors is greater than in all other Cases (the channels are open for larger values of the signal-noise ratio). The number of errors is different for Cases 1 and 4 for several reasons. For Case 1: the Data channel code is 31, the Slot format is 13 , the Spreading factor is 32 , Rx finger power is $[0-10]$ in decibels, i.e. in both channels the signals power is different. For Case 4: the Data channel code is 7, the Slot format is 15 , the Spreading factor is $8, \mathrm{Rx}$ finger power is $\left[\begin{array}{ll}0 & 0\end{array}\right]$ in decibels, i.e. the signals power in both channels is the same. This reason results in fewer errors for Case 4.

It is interesting that the errors in data transmission for Case 5 is much less than for Cases 1 and 4, despite the increase in the speed of the RPAS to $50 \mathrm{~km} / \mathrm{h}$. This is explained by the fact that for Case 5 the conditions of data

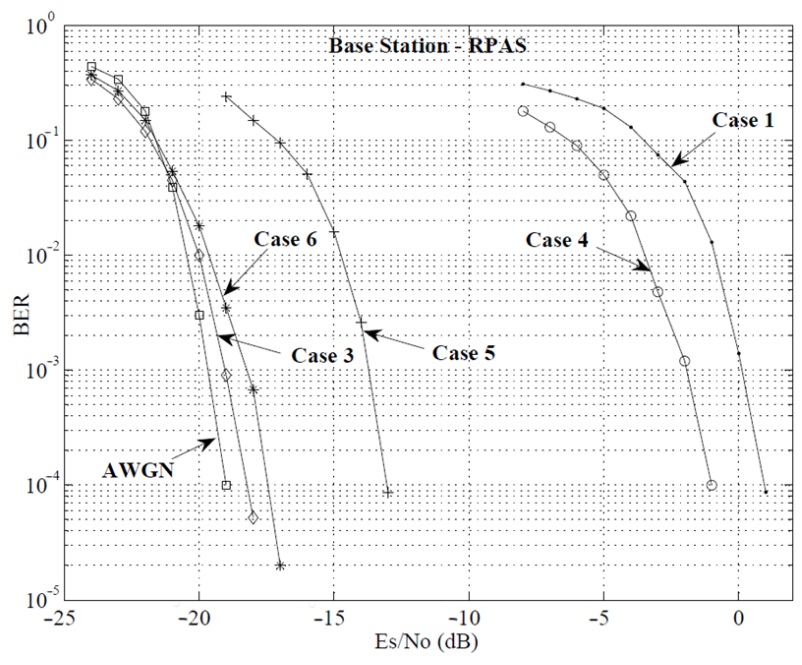

Figure 3. Dependencies of the BER on the signal-noise ratio for different RLoS channel types: BS antenna diameter $\approx 1.0 \mathrm{~m}$, RPAS antenna diameter $\approx 0.2 \mathrm{~m}$

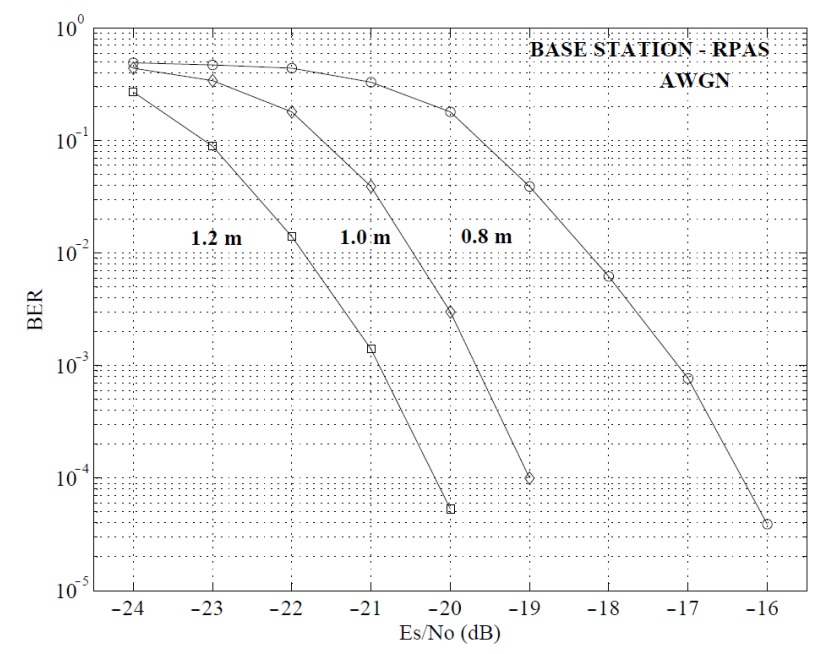

Figure 4. Dependencies of the signal-noise ratio on diameters of BS antennas: RLoS AWGN uplink, RPAS antenna diameter $\approx 0.2 \mathrm{~m}$ 


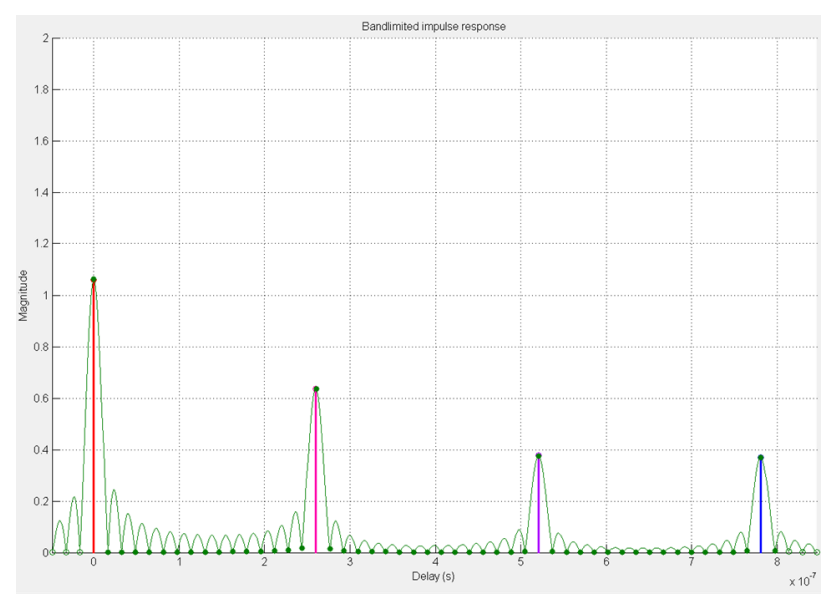

Figure 5. Channel bandlimited impulse response for Case 6 $(\mathrm{v}=250 \mathrm{~km} / \mathrm{h})$

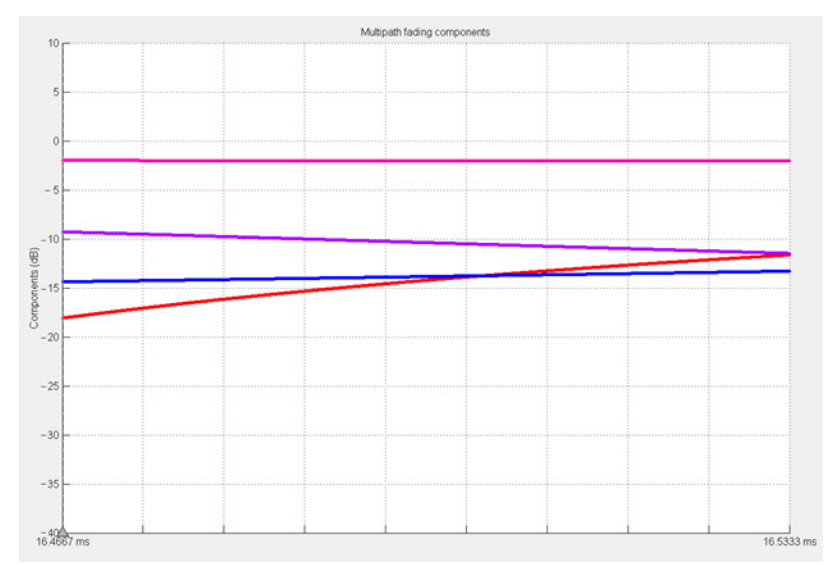

Figure 6. Multipath fading components for Case $6(\mathrm{v}=$ $250 \mathrm{~km} / \mathrm{h}$ )

transmission in the channel are different: the Data channel code is 7 , the Slot format is 15 , the Spreading factor is 8 , as in case 4 , but the signal powers are $[0-10]$, as in case 1 .

Of particular interest are Case $3(\mathrm{v}=120 \mathrm{~km} / \mathrm{h})$ and Case $6(\mathrm{v}=250 \mathrm{~km} / \mathrm{h})$, which are closest to the AWGN channel without fading (Figure 3). It would seem that the higher the RPAS speed, the more errors would have to be in the transmission of data. But it turns out that the use of Rake receiver and modification of the transmission conditions can reduce the BER to an acceptable level and even improve the situation in comparison with Cases 1, 4 and 5.

This clearly shows the main advantage of Rake Receiver, which improves the signal-noise ratio $\mathrm{E}_{s} / \mathrm{N}_{0}$. This improvement is observed in environments with a large number of multipath channels, and as can be seen from Figure 3, with significant speeds of the RPAS movement. The number of bit errors increases (compare Case 3 and Case 6 in Figure 3) with an increase in the RPAS speed under the same conditions of signals propagation and reception.

There is a problem in choosing the sizes of the BS and RPAS antennas for efficient and reliable data transmission. It is necessary to optimize the antenna parameters for se- lection the suitable size. Such justification and parameters choice are possible using the BER dependence on the signal-noise ratio (Figure 4). The simplest case of a nonfading AWGN channel was considered for one value of the RPAS antennas diameter. Decrease or increase within $20 \%$ in the BS antenna diameter significantly affects the operation of the communication channel.

When simulating a communication channel, it is possible to visualize a number of characteristics, including the impulse response of the signal (Figure 5) and the components of the multipath fading (Figure 6). Such characteristics were obtained and analyzed for all the cases listed in Table 1. At low RPAS speeds the impulse response and components of the multipath fading either do not change at all with time or change very slowly. At high speeds, these characteristics change quickly enough. Peaks presented in Figure 5 constantly change relative heights, and their power levels constantly "float" in time (Figure 6).

\section{BS - Satellite - RPAS communication channels simulation}

The model of "Base Station-to-Satellite-to-RPAS" link was designed on the base of "Base Station-to-RPAS" link (Figure 1) by adding the Satellite Transponder (Figure 7). The Satellite Transponder includes an antenna amplifier, a lowfrequency amplifier with noise temperature, a phase-frequency shift unit and an antenna amplifier that transmits data to the RPAS. Phase and frequency shifts were chosen to be zero. The Satellite Transponder antennas gains were taken 7.8 (an antenna diameter $\approx 1.0 \mathrm{~m}$ at $1 \mathrm{GHz}$ ).

In the amplifier block, there is a linear mode and modes for modelling nonlinearities - Cubic, Polynomial, Hyperbolic Tangent, Saleh, Ghorbani and Rapp, which are described in paper (Kharchenko, Grekhov, \& Ali, 2016). Power amplifiers are an important component in modern communications systems, providing the transmit signal levels needed to overcome the loss between the transmitter and receiver. The linear method is implemented by a Gain block (with a linear gain $10 \mathrm{~dB}$ ). All five subsystems for the nonlinear method options apply a memoryless nonlinearity to the complex baseband input signal. The dependence of the BER on the ratio $E_{s} / N_{0}$ for AWGN satellite channel with transponder nonlinearities is shown in Figure 8. For comparison, the case of a linear transponder amplifier is shown too. As can be seen from Figure 8, the number of errors for the linear amplifier is the smallest. The appearance of the nonlinearity leads to a significant deterioration in the quality of data transmission through the satellite communication channel. The channel turns out to be open only with an increase of about twice the value of the ratio $\mathrm{E}_{\mathrm{s}} / \mathrm{N}_{0}$ in comparison with the linear amplifier (Figure 8). The case of the Saleh model with a small nonlinearity gives the least number of bit errors. This model simulates the operation of a traveling wave lamp.

The dependencies of the BER on the signal-to-noise ratio $\mathrm{E}_{\mathrm{s}} / \mathrm{N}_{0}$ for different diameters of $\mathrm{BS}$ antennas were given in Figure 4 for the direct link between the base sta- 


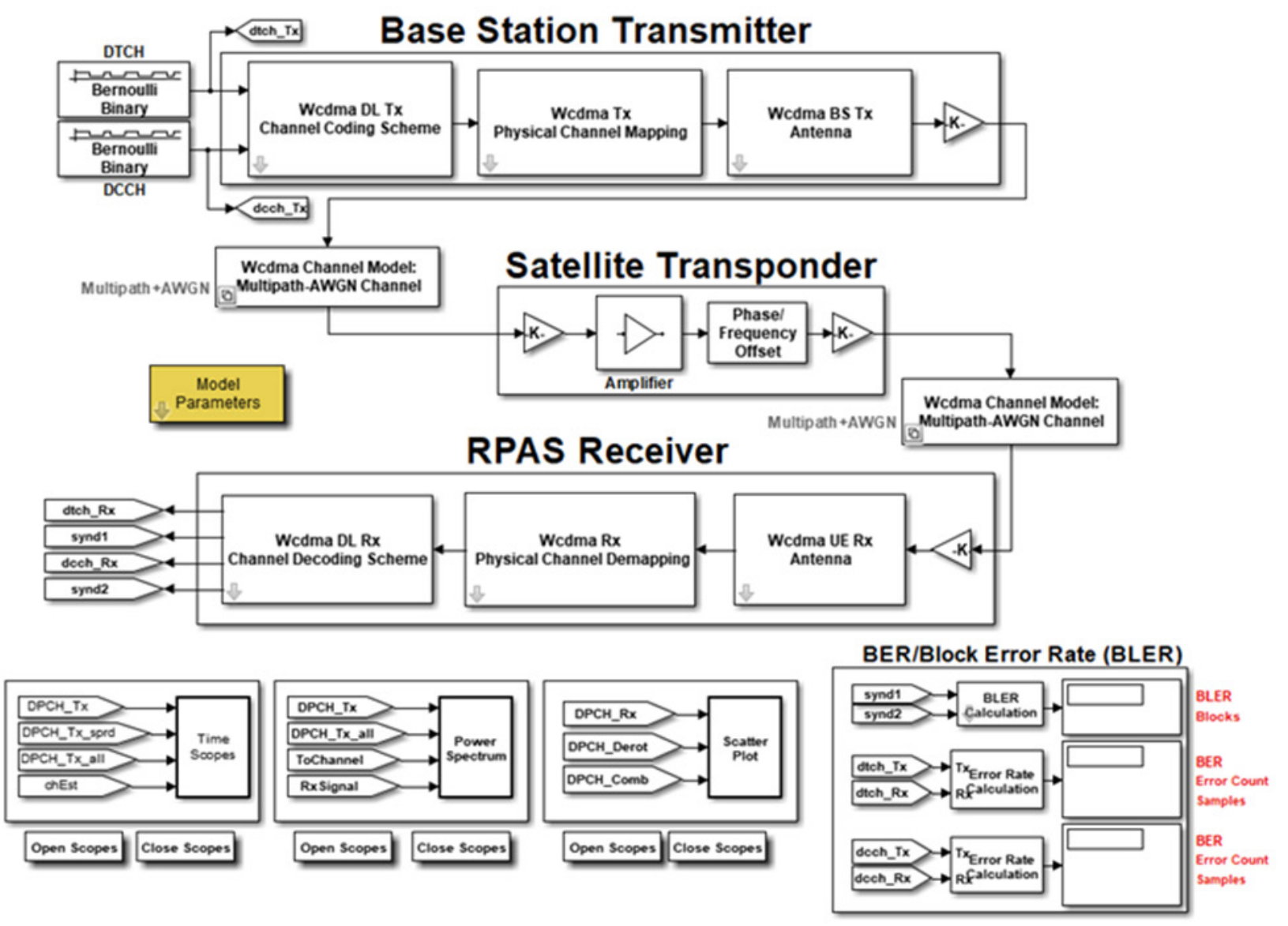

Figure 7. "Base Station-to-Satellite-to-RPAS” link

tion and the RPAS. However, ordinary RPASs that are controlled via RLoS have a significant drawback - they cannot fly far from the BS control center, because they will immediately lose the signal. Satellite communication technologies are designed to overcome this drawback. Moreover, the largest RPASs, such as Predator, Reaper and Global Hawk, send most of the video information they collect via communication satellites. The amount of information transmitted through the satellites from the RPAS can be several times greater than the capacity of the US military communications satellites. Such data transmission uses information compression, as well as sending several data streams simultaneously (multiplexing communication channels). Special software of RPAS cameras can also be used, which can determine what should be sent to the operator and what can be stored for some time on the RPAS itself. Only individual frames may be broadcast, indicating that it may be useful.

Modified versions of large multi-purpose Heron and Eitan RPASs, which are equipped with a satellite control system and which can travel more than 1000 kilometers, are created in Israel. However, they are not "tied" to the direct radio signal of the ground center or the air repeater. The latest developments allow controlling the RPAS via satellite communications, which greatly expands its tactical capabilities.
A simulation of satellite communication channel with different sizes of the BS antenna was carried out for BRLoS AWGN uplink and downlink. Dependencies of the BER on diameters of BS antennas are given in Figure 9. Small Saleh transponder nonlinearity was considered. Qualitatively, the effect of changing the BS antenna diameter is similar to that is shown in Figure 4 for RLoS communication channel. However, in the case of a satellite communication channel with a small non-linearity, the channel is open for larger values of the signal-to-noise ratio $\mathrm{E}_{\mathrm{s}} / \mathrm{N}_{0}$.

\section{Conclusions}

RPAS applications are very promising and useful, but many technical and organizational challenges have to be addressed in order to exploit drones successfully. Among the crucial issues needed to be solved, designers and researchers name the following: navigation, guidance and control, reliable communication between drones and the ground control station, good collaboration between drones in swarms, regulatory framework, and safe operations in non-segregated environment. Moreover, now the efficiency and economy of RPASs using comes to the forefront. Ensuring reliable and efficient communications between RPAS units on the line of sight and via satellites plays an 


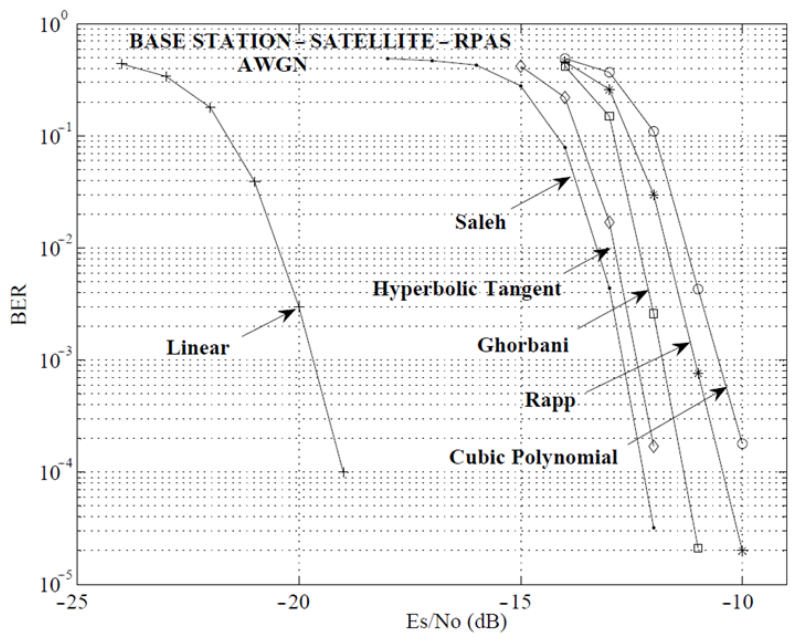

Figure 8. Dependencies of the BER on the signal-noise ratio for different levels of satellite transponder nonlinearity: BRLoS

AWGN uplink and downlink, noise temperature $290 \mathrm{~K}$;

BS antenna diameter $\approx 1.0 \mathrm{~m}$, satellite antennas diameters $\approx$ $1.0 \mathrm{~m}$, RPAS antenna diameter $\approx 0.2 \mathrm{~m}$, satellite transponder amplifier linear gain $10 \mathrm{~dB}$

important role in achieving the required performance and safety requirements. Issues of more efficient data transfer between RPAS and ground infrastructure, types of technologies used, conditions for reliable data exchange are of paramount importance. Now these problems have not yet been fully considered and theoretically not properly studied. At the same time, understanding the behavior of the system in critical conditions is crucial, since problems can be solved by simply switching to other modes or using other technologies. That is why modeling the RPAS channel for communication with the ground control station can significantly reduce the cost of deploying new systems. This work is devoted to the theoretical study of Space-Air-Ground Integrated Networks (SAGINs) based on WCDMA 3GPP Standard. The article develops the basics for obtaining numerical information about RPAS channel characteristics that is necessary for predicting its behavior. Currently, there are no such characteristics in the literature for communication channels with fading and the RPAS movement. All results listed below were obtained for the first time.

1. RPAS channel models with direct (RLoS) and satellite (BRLoS) links based on WCDMA 3GPP Standard were designed (Figure 1, Figure 7).

2. The quantitative characteristics of the fading communication channel (Table 1) were obtained for static RPAS and for its travel speeds of $3 \mathrm{~km} / \mathrm{h}$, $120 \mathrm{~km} / \mathrm{h}$ and $250 \mathrm{~km} / \mathrm{h}$ relative to the base station (Figure 3).

3. The dependencies of the BER on the antenna diameters of the base station for direct (Figure 4) and satellite (Figure 9) connections are obtained taking into account the non-linearity of the satellite transmitter.

4. The behavior of multipath fading components was analyzed (Figure 6) for RPAS speed $250 \mathrm{~km} / \mathrm{h}$.

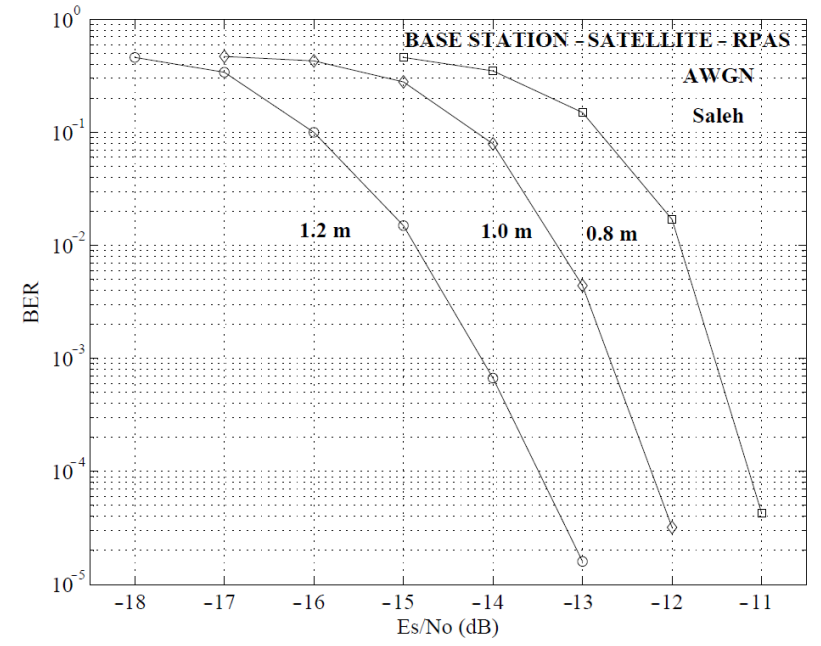

Figure 9. Dependencies of the BER on diameters of BS antenna: BRLoS AWGN uplink and downlink, noise temperature $290 \mathrm{~K}$; satellite antennas diameters $\approx 1.0 \mathrm{~m}$, RPAS antenna diameter $\approx 0.2 \mathrm{~m}$, Saleh transponder nonlinearity

5. The nonlinearity effect of the satellite transponder amplifier has been studied for five types of nonlinearity and a comparison has been made with a linear amplifier (Figure 8).

The significance of obtained results lies in the fact that this knowledge can help identify problems in the early stages of RPAS channel design, taking into account its movement. Understanding the behavior of RPAS data transmission channel in critical modes allows correctly configure real SAGIN systems. This minimizes deployment errors and reduces development time.

\section{References}

Bing, L. (2017). Study on modeling of communication channel of UAV. International Congress of Information and Communication Technology (ICICT 2017). Procedia Computer Science, 107, 550-557. https://doi.org/10.1016/j.procs.2017.03.129

Cao, X., Yang, P., Alzenad, M., Xi, X., \& Wu, D. (2018). Airborne communication networks: a survey. IEEE Journal on Selected Areas in Communications, 36(9), 1907-1926. https://doi.org/10.1109/JSAC.2018.2864423

Dong, Y., Hassan, Md. Z., Cheng, J., Hossain, Md. J., \& Leung, V. C. M. (2018). An edge computing empowered radio access network with UAV-mounted FSO fronthaul and backhaul: key challenges and approaches. IEEE Wireless Communications, 25(3), 154-160. https://doi.org/10.1109/MWC.2018.1700419

Fotouhi, A., et al. (2019). Survey on UAV cellular communications: practical aspects, standardization advancements, regulation, and security challenges. IEEE Communications Surveys \& Tutorials, 21(4), 3417-3442. https://doi.org/10.1109/COMST.2019.2906228

Grekhov, A. (2019). Recent advances in satellite aeronautical communications modeling (313 p.). IGI Global, USA. https://doi.org/10.4018/978-1-5225-8214-4

Grekhov, A., Kondratiuk, V., \& Ilnytska, S. (2018). Nonlinearities impact on satellite RPAS communication in clusters. Global Journal of Researches in Engineering (F), XVIII(I), 5-12. 
Grekhov, A., Kondratiuk, V., Ilnytska, S., Vyshnyakova, Y., Kondratiuk, M., \& Trykoz, V. (2019). Satellite traffic simulation for RPAS swarms. In Proceedings of the 2019 IEEE 5th International Conference Actual Problems of Unmanned Aerial Vehicles Developments (APUAVD) (pp. 265-270). Kyiv, Ukraine. https://doi.org/10.1109/APUAVD47061.2019.8943881

Gupta, L., Jain, R., \& Vaszkun, G. (2015). Survey of important issues in UAV communication networks. IEEE Communications Surveys and Tutorials, $P P(99), 1-32$.

Hanscom, A., \& Bedford, M. (2016). Unmanned aircraft system service demand 2015-2035, literature review \& projections of future usage. Research and Innovative Technology Administration, U.S. Department of Transportation, Washington, DC, USA, Technical Report DOT-VNTSC-DoD-13-01.

Jawhar, I., Mohamed, N., Al-Jaroodi, J., Agrawal, P. D., \& Zhang, S. (2017). Communication and networking of UAVbased systems: classification and associated architectures. Journal of Network and Computer Applications, 84, 93-108. https://doi.org/10.1016/j.jnca.2017.02.008

Khan, M. A., Qureshi, I. M., \& Khanzada, F. (2019). A hybrid communication scheme for efficient and low-cost deployment of future Flying Ad-Hoc Network (FANET). Drones, 3(16), 2-22. https://doi.org/10.3390/drones3010016

Khuwaja, A., Chen, Y., Zhao, N., Alouini, M., \& Dobbins, P. (2018). A survey of channel modeling for UAV communications. IEEE Communications Surveys \& Tutorials, 20(4), 2804-2821. https://doi.org/10.1109/COMST.2018.2856587

Kutsenko, O., Ilnytska, S., Kondratyuk, V., \& Konin, V. (2017). Unmanned aerial vehicle position determination in GNSS landing system. In Proceedings of the 2017 IEEE 4th International Conference Actual Problems of Unmanned Aerial Vehicles Developments (APUAVD) (pp. 79-83). Kyiv, Ukraine. https://doi.org/10.1109/APUAVD.2017.8308781
Liu, J., Shi, Y., Fadlullah, Z., \& Kato, N. (2018). Space-air-ground integrated network: a survey. IEEE Communications Surveys \& Tutorials, 20(4), 2714-2741. https://doi.org/10.1109/COMST.2018.2841996

Marchese, M., Moheddine, A., \& Patrone, F. (2019). IoT and UAV integration in 5G hybrid terrestrial-satellite networks. Sensors, 19(3704), 2-19. https://doi.org/10.3390/s19173704

MATLAB. (2016). Example "WCDMA End-to-End Physical Layer". Mozaffari, M., Saad, W., Bennis, M., Nam, Y. H., \& Debbah, M. (2019). A tutorial on UAVs for wireless networks: applications, challenges, and open problems. arXiv preprint arXiv:1803.00680. https://doi.org/10.1109/COMST.2019.2902862

Sharma, V. (2019). Advances in drone communications, state-ofthe-art and architectures. Drones, 3(21), 1-2. https://doi.org/10.3390/drones3010021

Yan, C., Fu, L., Zhang, J., \& Wang, J. (2019). A comprehensive survey on UAV communication channel modeling. IEEE Access, 4(1), 1-24. https://doi.org/10.1109/ACCESS.2019.2933173

Zeng, Y., Zhang, R., \& Lim, J. (2016a). Wireless communications with unmanned aerial vehicles: opportunities and challenges. IEEE Communications Magazine, 54(5), 36-42. https://doi.org/10.1109/MCOM.2016.7470933

Zeng, Y., Zhang, R., \& Lim, J. (2016b). Throughput maximization for UAV-enabled mobile relaying systems. IEEE Transactions on Communications, 64(12), 4983-4996. https://doi.org/10.1109/TCOMM.2016.2611512

Zeng, Y., \& Zhang, R. (2017). Energy-efficient UAV communication with trajectory optimization. IEEE Transactions on Wireless Communications, 16(6), 3747-3760. https://doi.org/10.1109/TWC.2017.2688328 